Eine Vorlagepflicht nach Art. 267 Abs. 3 AEUV besteht ohnehin nicht, weil es sich vorliegend nicht um eine letztinstanzliche Entscheidung handelt.

$[\ldots]$

DOI: 10.1007/s00350-017-4685-y

\section{Anmerkung zu OLG Stuttgart, Urt. v. 2.3.2017 - 2 U 11/14 (LG Tübingen)}

\section{Kerrin Schillhorn}

Im Nachgang $\mathrm{zu}$ der Entscheidung des BGH vom 24.3.2016 - 1 ZR 263/14 -, in der die Grundlagen der Anwendung des europäischen Beihilferechts auf öffentliche Zuwendungen an Krankenhäuser in öffentlicher Trägerschaft anhand dieses konkreten Einzelfalls ausführlich behandelt wurden, hatte das OLG hier die vom BGH noch offen gelassene Frage zu prüfen, ob eine Begünstigung der Kreiskliniken im beihilferechtlichen Sinne vorlag. Dabei hat sich das OLG von dem sehr weiten Verständnis des EuGH in Bezug auf die gemeinschaftsrechtliche Auslegung der Beeinflussung des europäischen Wettbewerbs gelöst und sehr genau geprüft, ob die gewährten Zuwendungen den Handel zwischen Mitgliedstaaten tatsächlich beeinträchtigen (können). Die vorgenommene Prüfung und das gefundene Ergebnis, dass durch die gewährten Beihilfen ein gemeinschaftsrechtlicher Bezug zu dem Handel zwischen den Mitgliedstaaten nicht festgestellt werden könne, ist anhand der ausgeführten Umstände der Fallkonstellation ohne weiteres nachzuvollziehen. Es handelte sich um kommunale Krankenhäuser auf Kreisebene, die im Wesentlichen der Grundversorgung der umliegenden Bevölkerung dienten. Weder wurden medizinische Spezialangebote vorgehalten noch wiesen die beiden Krankenhäuser einen Einzugsbereich auf, der über den lokalen Bereich hinaus ging. Dieses Ergebnis scheint auf der Basis der geprüften Umstände des Einzelfalls plausibel und auch sinnvoll insoweit, als eine tatsächliche Auswirkung auf den Handel zwischen Mitgliedstaaten hierdurch wohl nicht ohne weiteres gesehen werden kann. Dies würde bedeuten, dass nicht eine grundsätzliche Wettbewerbsrelevanz im europäischen Kontext angenommen werden kann, nur weil der deutsche Gesundheitsmarkt grundsätzlich auch ausländischen Trägern offen stehen kann, sondern dass im Einzelfall konkret geprüft werden muss, ob tatsächlich ein Gemeinschaftsbezug der Leistungserbringung und seiner Vergütung besteht.

Neben den tatsächlichen Kriterien für die Prüfung der lokalen oder aber gemeinschaftsweiten Auswirkungen stellte das OLG auch auf die Besonderheit eines Unternehmens ab, das Dienstleistungen von allgemeinem öffentlichen Interesse erbringt. Für ein solches hielt das OLG, wie schon der BGH in seiner Entscheidung vom 24.3.2016 - 1 ZR 263/14 -, die hier streitgegenständlichen Krankenhäuser wegen ihrer gleichzeitigen Aufnahme in den Krankenhausplan des Landes und der landesrechtlichen Verpflichtung zur Sicherstellung der stationären Versorgung der Bevölkerung. Eine ähnliche Regelung wie $\$ 3$ Abs. 1 LKHG BW findet sich in fast jedem Landeskrankenhausgesetz. Im Ergebnis bedeutet dies, dass jedes Krankenhaus in kommunaler bzw. öffentlicher Trägerschaft, das in den Krankenhausplan des Landes aufgenom-

Rechtsanwältin Dr. iur. Kerrin Schillhorn, MIL,

Fachanwältin für Verwaltungsrecht und

Fachanwältin für Medizinrecht,

michels.pmks Rechtsanwälte Partnerschaft mbB

Hohenstaufenring 57, 50674 Köln, Deutschland men ist, ein solches Unternehmen darstellt, das besondere Dienstleistungen von allgemeinem öffentlichen Interesse übernimmt. Dabei findet eine Spezifizierung des Leistungsgeschehens im Einzelnen durch das OLG nicht statt. Zuzugeben ist sicherlich, dass die zur Verhinderung einer Versorgungslücke erforderlichen Kapazitäten nicht erst bei deren Eintritt geschaffen werden können, sondern permanent vorgehalten werden müssen. Aber eine Betrachtung des Unternehmens „kommunales Krankenhaus“ ohne dessen Disziplinenspektrum und dessen Spezialisierungen erscheint insoweit fragwürdig, als möglicherweise nicht alle Fachgebiete und Spezialisierungen des Krankenhauses auch tatsächlich den Sicherstellungsauftrag nach dem Landesrecht erfüllen.

Vielmehr wäre es hier erforderlich gewesen, eine umfassendere Prüfung des Leistungsspektrums im Hinblick auf die Sicherstellung der Versorgung der Bevölkerung durchzuführen. Seit längerer Zeit etabliert sich im Krankenhausplanungsrecht der Ansatz, dass insbesondere spezialisierte und medizinisch sehr aufwendige Behandlungen nicht flächendeckend in allen Krankenhäusern vorgehalten werden sollen, sondern insoweit eine gestufte Versorgung zwischen kleineren Krankenhäusern vor Ort und größeren und großen Krankenhäusern in Mittel- und Oberzentren erfolgen soll. Dieser Aspekt hätte in der Entscheidung mit berücksichtigt werden müssen, wenn der Sicherstellungsauftrag des Krankenhauses ein wesentliches Kriterium im Hinblick auf die beihilferechtliche Beurteilung von Zuwendungen an dieses Krankenhaus darstellt. Insgesamt erscheint die zwingende Verknüpfung zwischen Krankenhausplanungsrecht einerseits und dem abstrakten Sicherstellungsauftrag der kommunalen Krankenhäusern nach dem Landesrecht andererseits, die bereits durch den $\mathrm{BGH}$ vorgenommen wurde, nicht überzeugend. Zum einen bietet das Krankenhausrecht selbst das Instrument der Sicherstellungszuschläge, falls ein bedarfsnotwendiges Krankenhaus defizitär wird und die notwendigen Versorgungsleistungen nicht mehr kostendeckend anbieten kann. Zum anderen sind Zweifel angebracht, ob in Zeiten des bundesweit diskutierten Überhangs an Krankenhausbetten die Aufnahme in den Krankenhausplan als bedarfsgerechtes Krankenhaus die gleichen Anforderungen erfüllt wie sie an die Sicherstellung der Versorgung der Bevölkerung mit Krankenhausleistungen im Sinne des jeweiligen Landesrechts zu stellen sind. Es wird derzeit intensiv diskutiert, wie viele Krankenhausbetten überhaupt erforderlich sind und welche Disziplinen und Spezialisierungen an welchen Krankenhausstandorten überhaupt noch bedarfsgerecht sind. Vor diesem Hintergrund der aktuellen Entwicklungen in der Krankenhausplanung erscheint es keineswegs zwingend, die alleinige Aufnahme in den Krankenhausplan mit der allgemeinen Verpflichtung zur Sicherstellung nach Landesrecht so eng zu verknüpfen und daraus abzuleiten, dass das Krankenhaus als notwendige Institution der öffentlichen Daseinsvorsorge erforderlich ist und damit von den Anforderungen des europäischen Beihilferechts befreit ist.

Auch wenn dieses formale Kriterium sicherlich leichter $\mathrm{zu}$ handhaben ist als eine konkrete Prüfung der notwendigen Sicherstellung im Einzelfall, so verbleibt doch ein gewisser Zweifel, ob damit der inzwischen sehr vielfältigen Krankenhauslandschaft vor dem Hintergrund des freiheitlichen Wettbewerbsrechts auf europäischer Ebene tatsächlich ausreichend Rechnung getragen ist. Es bleibt abzuwarten, ob diese Entscheidungen, sowohl des BGH als auch des OLG, dauerhaft Bestand haben werden. So dürfte zu erwarten sein, dass die Privatkliniken sich mit diesem Ergebnis nicht zufrieden geben und die Fragestellung erneut zur gerichtlichen Überprüfung bringen, um gegebenenfalls auch eine konkrete Entscheidung des EuGH zu dieser Thematik zu erhalten. 